\title{
Trombose de artéria mesentérica superior em paciente portadora de provável coagulopatia
}

\author{
Tássia Callai ${ }^{1}$; Jéssica Francine Wichmann²; Nicolas Lauxen \\ Konrad 3 ; Angelina Bopp Nunes ${ }^{4}$; Marina Fernandes Bianchi ${ }^{5}$; \\ Daniela Teixeira Borges ${ }^{6}$; Sérgio Henrique Prezzi ${ }^{7}$
}

\section{Resumo}

Introdução: A isquemia mesentérica é uma rara condição médica que representa $0,1 \%$ de todas as admissões hospitalares, com altas taxas de mortalidade que variam de $24 \%$ a $94 \%$. Ocorre geralmente entre a quinta e sexta década de vida, e a incidência aumenta com idade. A apresentação típica é uma pessoa idosa com múltiplas comorbidades, queixando-se dor abdominal grave desproporcional ao exame físico. Objetivo: Objetiva-se relatar o caso de trombose proximal de artéria mesentérica superior e extensa necrose de intestino delgado em paciente do sexo feminino com coagulopatia provável por associação de uso de anticoncepcional oral com tabagismo. Descrição do caso: Paciente L.A.H. feminina, 40 anos, branca, solteira, encaminhada ao hospital com dor abdominal súbita, lacerante,

\footnotetext{
${ }^{1}$ Acadêmica do curso de Medicina da Universidade de Santa Cruz do Sul (UNISC). tassiacallai@hotmail.com

2 Acadêmica do curso de Medicina da Universidade de Santa Cruz do Sul (UNISC). jessicafwichmann@gmail.com

${ }^{3}$ Acadêmico do curso de Medicina da Unidade Integrada Vale do Taquari de Ensino Superior (UNIVATES).nicolaskonrad@hotmail.com

${ }^{4}$ Acadêmica do curso de Medicina da Universidade de Santa Cruz do Sul (UNISC). angelina_bopp_nunes@hotmail.com

5 Acadêmica do curso de Medicina da Universidade de Santa Cruz do Sul (UNISC). maarina.bianchi@hotmail.com

${ }^{6}$ Médica de Família e Comunidade, Preceptora da Residência em Medicina de Família e Comunidade (UNISC/HSC), Docente da Graduação em Medicina (UNISC), Prof. Coordenadora da Liga de Medicina de Família e Comunidade (UNISC) e do Internato em Medicina de Família e Comunidade (UNISC/HSC), Tutora PET-Saúde Vigilância (UNISC), Doutoranda em Saúde Coletiva (UNISINOS), Mestre em Envelhecimento Humano (UPF), Especialista em Medicina de Família e Comunidade (AMB - SP), Especialista em Saúde da Família (UFCSPA/UNA-SUS), Especialista em Saúde Coletiva (UPF), Especialista em Humanização da Atenção e da Gestão do SUS (UFRGS), Graduação em Medicina (UPF, 2005)

${ }^{7}$ Preceptor de equipe do Serviço de Medicina Interna do Hospital de Clínicas de Porto Alegre, especialista em Terapia Intensiva, Cardiologia e Nefrologia. sprezzi@portoweb.com.br
} 
associado a náuseas e hematêmese.Realizado diagnóstico de isquemia mesentérica aguda. A ultrassonografia abdominal evidenciou íleo adinâmico, porém descartou processo inflamatório íleocecal. Angiotomografia evidenciou trombose proximal de artéria mesentérica superior. Realizada laparotomia exploradora, sendo evidenciada extensa necrose de delgado por isquemia do terço proximal da artéria mesentérica, sendo necessário ressecção intestinal extensa. Paciente previamente hígida, tinha história pessoal e familiar negativas para fenômenos tromboembólicos. Fazia uso de anticoncepcional oral. Tabagista 9 maços/ ano à 18 anos. Etilista social ativa. Laboratoriais: VDRL, anticorpos anti T. pallidum, HBSAg, Monoteste, ANTI-HCV não reagentes, teste de falcização negativo, AC Anti-cardiolipina IgG 1,5 GLP, AC Anticardiolipina IgM 4,7 MLP, pesquisa de auto-ancorpos (FAN e citoplasmáticos) negativo, Anti-coagulante Lúpico 1,13, fator reumatoide $<10,6$ (r: $<40 \mathrm{UL} / \mathrm{ml}$ ), Antirombina III 80\% (r:83-128\%), contagem de plaquetas 326.000/ $\mathrm{LL}$, fibrinogênio $426 \mathrm{mg} / \mathrm{dL}$, p-Anca e c-Anca negativos. Homocisteinemia negativo. KTTP: 25seg; TP: 15,5seg. Pesquisa da mutação R506Q do fator V de Leiden negativo; Pesquisa da mutação da protrombina negativo. Conclusão: $\mathrm{O}$ estudo realizado com base na história clínica da paciente sugere um caso de trombose arterial secundária a coagulopatia. Observou-se relação entre a isquemia mesentérica causada pela trombose de artéria mesentérica superior com o uso de anticoncepcional oral associado ao tabagismo, mostrado na literatura como agente responsável por propiciar ou agravar a ocorrência de coagulopatias. 


\section{Introdução}

A isquemia mesentérica (IM) é uma rara condição médica que representa $0,1 \%$ de todas as admissões hospitalares, com altas taxas de mortalidade que variam de $24 \%-94 \%$. A IM inclui fornecimento inadequado de sangue, lesão inflamatória e, eventualmente, necrose da parede do intestino.(MASTORAKI, 2016)

Ocorre geralmente entre a quinta e sexta década de vida, e a incidência aumenta com idade. A localização mais comum corresponde à raiz do mesentério envolvendo o intestino delgado (90\%) embora às vezes possa envolver a mesocolo, região peripancreática, omento, retroperitônio e pelve. (SOLANAS; HUGUET, 2016)

Esta condição continua a desafiar até mesmo os médicos mais experientes devido a seus sintomas vagos e inespecíficos, resultando em diagnósticos tardios e taxas de sobrevivência historicamente sombrias. A apresentação típica é uma pessoa idosa com múltiplas comorbidades, queixando-se dor abdominal grave desproporcional ao exame físico. A dor é constante, difusa, não localizada ou periumbilical, e sintomas como vómitos, diarreia, distensão abdominal e enterorragia ocorrem com frequência, porém não são universais.

A idade avançada e as múltiplas comorbidades normalmente presentes nesses pacientes com IM significam, apesar de uma identificação adequada, uma mortalidade superior a $50 \%$ em séries recentes (SCHOLZ, 2015). Os danos ao intestino são proporcionais ao tempo e à diminuição do fluxo sanguíneo mesentérico e podem variar de lesões mínimas, devido a isquemia reversível, até lesão transmural, com necrose e posterior perfuração.(REGINELLI et al., 2013; SERRACANT BARRERA et al., 2015)

Suas causas variam de tromboembolia arterial à trombose venosa mesentérica (TVM) e causas não- oclusivos, tais como hipoperfusão devido ao baixo débito cardíaco ou vasoconstrição arterial mesentérica. Outras causas incluem a displasia fibromuscular , vasculite, arterite de Takayasu, malignidade e radiação. (MASTORAKI, 2016; SCHOLZ, 2015). Distúrbios de coagulação também fazem parte do diagnóstico diferencial quando investigada a causa da IM.

Enquanto os estudos laboratoriais ou radiografias simples de abdome não são acurados para o diagnóstico, a tomografia computadorizada (TC) e angiotomografia contribuem para o diagnóstico diferencial e gestão da MI. A ultrassonografia (US) e angiografia por ressonância magnética (MRA) são considerados também de grande importância. $O$ primeiro para exclusão de outras causas de abdome agudo, e o segundo como padrãoouro no diagnóstico de IM. A abordagem terapêutica de IM inclui tanto o manejo clínico e medicamentoso quanto a intervenção cirúrgica, quando necessária.(DURAN et al., 2015; REGINELLI et al., 2013).

O presente trabalho tem como objetivo relatar o caso de trombose proximal de artéria mesentérica superior e extensa necrose de intestino delgado em paciente do sexo 
feminino com coagulopatia provável por associação de uso de anticoncepcional oral com tabagismo.

\section{Relato do caso}

Paciente L.A.H. feminina, 40 anos, branca, solteira, encaminhada ao hospital com dor abdominal súbita, lacerante, associado a náuseas e hematêmese. Realizado diagnóstico de isquemia mesentérica aguda. Paciente transferida para hospital de referência para a realização de cirurgia. Na chegada paciente encontrava-se em regular estado geral, sob ventilação mecânica, sedada, hemodinamicamente estável. A ultrassonografia abdominal evidenciou íleo adinâmico, porém descartou processo inflamatório íleocecal. Angiotomografia evidenciou trombose proximal de artéria mesentérica superior.

Realizada laparotomia exploradora, sendo evidenciada extensa necrose de delgado por isquemia do terço proximal da artéria mesentérica, sendo necessário ressecção intestinal extensa e a $25 \mathrm{~cm}$ do ângulo de Treitz até $25 \mathrm{~cm}$ antes da válvula ileocecal em 27/01/16, restando $50 \mathrm{~cm}$ de intestino delgado.

Paciente previamente hígida, tinha história pessoal e familiar negativas para fenômenos tromboembólicos. Fazia uso de anticoncepcional oral, Omeprazol, Bromoprida e Lorazepam. Tabagista 9 maços/ ano à 18 anos. Etilista social ativa.

Durante internação realizou investigação diagnóstica. Realizou ecocardiograma transeofagico que identificou presença de trombo móvel em aorta torácica descendente logo após à emergência da subclávia esquerda, medindo medindo aproximadamente 4,8 $\mathrm{mm}$ x 9,0 mm de dimensões transversas e 22,1 $\mathrm{mm}$ de extensão longitudinal desde a parede do vaso, Presença de trombo móvel em originário da junção da veia cava superior com o átrio direito, medindo aproximadamente $35 \mathrm{~mm}$ de extensão. Presença de forame oval patente. No seguimento, diagnóstico tomográfico de evolução de trombos, progredindo com infarto esplênico e renal 22/02/2016 mesmo sob vigência de anticoagulação à pleno com HBPM.

Laboratoriais: VDRL, anticorpos anti T. pallidum, HBSAg, Monoteste, ANTI-HCV não reagente, teste de falcização negativo, AC Anti-cardiolipina IgG 1,5 GLP, AC Anticardiolipina IgM 4,7 MLP, pesquisa de auto-ancorpos (FAN e citoplasmáticos) negativo, Anti-coagulante Lúpico 1,13, fator reumatoide <10,6 (r:<40UL/ml), Antirombina III 80\% (r:83-128\%), contagem de plaquetas 326.000/ $\mu \mathrm{L}$, fibrinogênio 426 $\mathrm{mg} / \mathrm{dL}$, p-Anca e c-Anca negativos. Homocisteinemia negativo. KTTP: 25seg; TP: 15,5seg. Pesquisa da mutação R506Q do fator V de Leiden negativo; Pesquisa da mutação da protrombina negativo. Teste de deficiência de proteína $\mathrm{C}$ e $\mathrm{S}$ não foram realizados devido ao início da anticoagulação.

Optou-se por colocação de endoprótese nano em aorta descendente em 02/03/2016 na tentativa de impedir novas embolizações. 
Após extensa investigação concluiu-se distúrbio de hipercoagulabilidade devido a associação de tabagismo e uso de anticoncepcional oral.

\section{Discussão}

É estimado que a maioria dos casos de isquemia intestinal (65\%) são causados por embolia arterial ou trombose com insuficiência no fluxo sanguíneo da artéria mesentérica superior, afetando a totalidade ou porções do intestino delgado e cólon direito. Apesar de ser uma doença de etiologia desconhecida, já são descritos uma série de fatores causais, tais como trauma ou cirurgia previa abdominal. Outras causas menos prevalentes incluem doença arterial, autoimunidade, infecções, abuso de drogas, lesões térmicas ou químicas, vasculite e reações pancreatite associados a outros fatores propiciantes, como: doença arterial coronária, cirrose, aneurisma da aorta abdominal, ulceração péptica, ascite quilosa e tabaco.

O tabagismo propicia a ocorrência de coagulopatias, visto que a nicotina estimula a liberação de catecolaminas, estimula as lesões no endotélio arterial e promove a aterogênese. Radicais livres e compostos aromáticos diminuem a síntese de óxido nítrico no endotélio, prejudicando o relaxamento dependente do endotélio nas artérias. Desta forma, os tabagistas apresentam alteração tanto no balanço vascular biológico propiciando a trombogênese (efeitos nas plaquetas e fatores de coagulação), quanto no controle do tônus vascular, favorecendo a vasoconstrição. (NUNES; MARIA; WILKEN, 2012).

Além do tabagismo, o uso de contraceptivos orais está associado a um risco aumentado de doenças tromboembólicas devido a sua ação pró-coagulante e de aumento da atividade fibrinolítica e dos inibidores naturais da coagulação (FERREIRA; FRANCESCHINI; TOLOI, 2000), sendo considerados, atualmente, como fator associado a dezenas de casos de trombose mesentérica (SIMÃO; NADAI; GIACON; LOPES, 2006).

Assim, os estados de hipercoagulabilidade e as trombofilias, que podem estar associados ao uso concomitante de contraceptivos orais e tabagismo, podem determinar a formação de trombos e êmbolos.

A fim de investigar os casos de hipercoagulabilidade, em pacientes sem fatores de risco para outras comorbidade, devemos avaliar, além do tempo de protrombina e tromboplastina parcial ativada, devem ser avaliados submetidos a exames com vistas ao diagnóstico de trombofilias: Deficiência de proteína $\mathrm{C}, \mathrm{S}$ e antitrombina III, anormalidades no anticoagulante lúpico e anticorpo anticardiolipina.

A IM aguda é uma emergência abdominal rara, que usualmente requer extensa ressecção intestinal e apresenta alta taxa de mortalidade (ROMANO et al., 2011). 
O tratamento é baseado na revascularização do intestino, e deve ser realizado até 6 a 8 horas após o início dos sintomas, quando a isquemia ainda se apresenta reversível. (DURAN et al., 2015; SERRACANT BARRERA et al., 2015)

Quando há segmentos intestinais comprometidos, além da ressecção das alças inviáveis, a revascularização é importante no sentido de evitar a progressão da necrose. Nas fases tardias de infarto maciço, a revascularização não é indicada. Além da pequena possibilidade de minimizar a ressecção, favorece a reabsorção de substâncias produzidas pela lise celular e contaminação bacteriana capazes de produzir diminuição do volume plasmático, distúrbios eletrolíticos, hemorragia intestinal e colapso circulatório - é a síndrome de reperfusão (VIRGINI-MAGALHÃES; MAYALL, [s.d.]).

\section{Conclusão}

A isquemia mesentérica representa uma emergência cirúrgica incomum que necessita de diagnóstico precoce a fim de gerar opções terapêuticas que possam reduzir a morbimortalidade dos pacientes.

O estudo realizado com base na história clínica da paciente sugere um caso de trombose arterial secundária a coagulopatia. Observou-se relação entre a isquemia mesentérica causada pela trombose de artéria mesentérica superior com o uso de anticoncepcional oral associado ao tabagismo, mostrado na literatura como agente responsável por propiciar ou agravar a ocorrência de coagulopatias.

Produtos encontrados no cigarro, como a nicotina, induzem estado protrombótico, através da ativação plaquetária, e ao interagir com os anticoncepcionais orais causam um aumento do risco de eventos trombogênicos, indicando uma contribuição importante para a ocorrência da comorbidade da paciente.

A combinação de diagnóstico precoce, métodos de imagem e tratamento através da ressecção do intestino necrosado por isquemia do terço proximal da artéria mesentérica foi essencial para a melhora do prognóstico da paciente em questão.

\section{Referências}

DURAN, M. et al. The importance of open emergency surgery in the treatment of acute mesenteric ischemia. World journal of emergency surgery, v. 10, p. 45, 2015.

MASTORAKI, A. Mesenteric ischemia: Pathogenesis and challenging diagnostic and therapeutic modalities. World Journal of Gastrointestinal Pathophysiology, v. 7, n. 1, p. $125,2016$. 
REGINELLI, A et al. Intestinal Ischemia: US-CT findings correlations. Critical ultrasound journal, v. 5 Suppl 1, n. Suppl 1, p. S7, 2013.

SCHOLZ, F. J. Mesenteric Ischemia. Critical Care Clinics, 2015.

SERRACANT BARRERA, A. et al. Acute mesenteric ischemia: Utility of endovascular techniques. Cirugia espanola, p. 1-6, 2015.

SOlANAS, L. L.; HUGUET, M. M. Enfermedades del mesenterio. Procesos inflamatorios. Patología vascular. Isquemia mesentérica. Medicine - Programa de Formación Médica Continuada Acreditado, v. 12, n. 4, p. 178-188, 2016.

AĞAOĞLU, N. et al. Prevalence of prothrombotic abnormalities in patients with acute mesenteric ischemia. World Journal of Surgery, v. 29, n. 9, p. 1135-1138, 2005.

BJÖRCK, M. et al. Revascularization of the superior mesenteric artery after acute thromboembolic occlusion. British Journal of Surgery, v. 89, n. 7, p. 923-927, 2002.

Brito CJ, editor Cirurgia Vascular, endovascular e angiologia. $2^{\mathrm{a}}$ ed. Rio de Janeiro: Revinter. 2007. p. 943-69.

FERREIRA, A. C. P.; FRANCESCHINI, S. A; TOLOI, M. R. T. Efeitos do contraceptivo oral contendo $20 \mu \mathrm{g}$ de etinilestradiol e $150 \mu \mathrm{g}$ de desogestrel sobre os sistemas de coagulação e fibrinólise. v. 22, n. 2, p. 77-87, 2000.

KOZUCH, P. L.; BRANDT, L. J. Review article: Diagnosis and management of mesenteric ischaemia with an emphasis on pharmacotherapy. Alimentary Pharmacology and Therapeutics, v. 21, n. 3, p. 201-215, 2005.

NUNES, R.; MARIA, A.; WILKEN, O. Processo Inflamatório Na Aterosclerose. p. 53-69, 2012.

ROMANO, N. et al. Acute thrombosis of the superior mesenteric artery in a 39year-old woman with protein-S deficiency: a case report. Journal of medical case reports, v. 5, n. 1, p. 17, 2011.

VIRGINI-MAGALHÃES, C. E.; MAYALL, M. R. I Squemia M Esentérica. p. 70-80, [s.d.].

SIMÃO, J.L., et al. Uso de contraceptivos orais induzindo trombose mesentérica. 
Rev. bras. hematol. hemoter. 2008;30(1):75-77 . 